\title{
Improving Balance through Virtual Reality and Physical Therapy Integration
}

\author{
Ben Joseph S. Esguerra, Kristen Johnson \\ University of St. Augustine for Health Sciences, St. Augustine, USA \\ Email: benesguerra@hotmail.com
}

How to cite this paper: Esguerra, B.J.S. and Johnson, K. (2017) Improving Balance through Virtual Reality and Physical Therapy Integration. International Journal of Clinical Medicine, 8, 322-337.

https://doi.org/10.4236/ijcm.2017.85030

Received: April 22, 2017

Accepted: May 24, 2017

Published: May 27, 2017

Copyright $\odot 2017$ by authors and Scientific Research Publishing Inc. This work is licensed under the Creative Commons Attribution International License (CC BY 4.0).

http://creativecommons.org/licenses/by/4.0/

\begin{abstract}
Background and Purpose: Virtual reality (VR) is an innovative technology that shows promise in the assistance of physical therapy (PT). This case report explores the use of virtual reality with a patient suffering from unilateral vestibular hypofunction (UVH). Case Description: The patient is a 50-year-old male who was referred to physical therapy following a motor vehicle accident. The patient was diagnosed with having an acute left UVH, accompanied by reports of dizziness, imbalance and gait disturbances which impaired him from his work in construction. Intervention: The patient was seen two to three times a week for 40-minute sessions along with an individualized home exercise program. Interventions included vestibular rehabilitation exercises, balance training, gait training, and VR. The goals of VR were to provide taskspecific simulations to improve postural balance, decrease anxiety through exposure therapy, and improve smooth eye pursuits to improve static balance. Outcomes: Outcomes used included subjective questionnaires such as the Activities-Specific Balance Confidence survey and the Dizziness Handicap Inventory (DHI) as well as functional tests like the Sensory Organization Test, Motor Control Test, and the Functional Gait Assessment (FGA). Outcome measures were performed at initial evaluation, at the 10th visit, and again at discharge. Notable improvements were seen on DHI and FGA scores. Conclusion: Dizziness, confidence, balance, and gait improved following vestibular rehabilitation combined with VR. Outcomes of this case suggest that virtual reality in conjunction with vestibular rehabilitation therapy is effective in improving deficits of unilateral vestibular hypofunction. Additionally, the use of VR in this case report suggests this can be an effective tool for intervention to facilitate patient-specific goals.
\end{abstract}

\section{Keywords}

Physical Therapy, Acute Unilateral Vestibular Hypofunction, Virtual Reality, Vestibular Rehabilitation 


\section{Introduction}

Modern technology and the advent of the internet have changed the structure and organization of the entire medical field. Technology has given the fragmented nature of health care more efficient ways to sort through the large volume of transactions within the system, a way to provide the need to integrate new scientific evidence into practice, organize other complex information management activities, and free the limitations of paper-based information management [1]. New systems and technologies have been implemented to ease the transfer of information from one person to another. From the widespread adoption of electronic medical records to advances in bio-medical engineering and technology, modern healthcare and its delivery methods are continually changing. Due to the continually expanding body of knowledge in health care, practitioners who rely on their memory and personal experience are obsolete [1]. Electronic medical records have increased the efficiency of clinical practice, reduced human errors, and increased the quality of care. The increased connectivity of the internet has given rise to telecommunication. Patients now have access to speak to any a medical professional through the simple press of a button. Practitioners are also able to create virtual conferences with other professionals across the globe. The age of information has continued to evolve into the technological revolution of the 21st century. Not only has technology allowed an improved way to share information, but it has brought forth inventions of improved diagnostic tools within health care. Some of these tools include radiographic images, angiograms, ultrasound, nerve conduction, electrocardiograms, and electroencephalograms, just to name a few. Technology is continuously growing and useful in everyday life. New technology has been integrated into healthcare and has created better access to medical records, exchange of data, visualization of the human body, and assistance with the evaluation and treatment of patients. A newer, revolutionary technology, virtual reality, has continued to show promise as a rehabilitative tool.

Virtual reality (VR) is defined as an artificial environment which is experienced through sensory stimuli provided by a computer and in which one's actions partially determine what happens in the environment [2]. Previously, VR technology had a patient placed onto a screen in which the patient would see themselves as an avatar within a virtual world. Today, we are able to immerse people into a virtual world where their movements are tracked within a $3 \mathrm{di}$ mensional plane where they are able to manipulate and maneuver within the virtual environment. Continuing advances in VR technology and system cost reductions have allowed this technology to be accessible to the masses. Most VR systems primarily deliver visual and auditory feedback [3]. Information is displayed by head-mounted displays, projections systems, or a flat screen of varying sizes. Special applications and accessories may be necessary to run with these display systems [3] [4]. VR allows for control, customization and freedom within a given environment.

The first health care applications of VR started in the early 90s by the need of 
medical staff to visualize complex medical data, particularly during surgery and for surgery planning. At this time, the surgery-related applications of VR fell under these categories: surgery training, surgery planning and augmented reality for surgery sessions in open surgery, endoscopy, and radiosurgery [5]. In the following years, VR applications were developed and broadened to include neuropsychological assessment and rehabilitation. Even with the ability of variance and customization of VR, the gap between the virtual experience and the complexity of real life was far too much.

With our advances in technology, VR has become the forefront and is considered one of the most innovative technologies and promises to have a considerable impact on rehabilitation [3]. New VR systems and applications have the ability to uniquely target a broad range of physical, psychological, and cognitive rehabilitation concerns [4]. Each application requires special software development tools to design an interactive simulated environment that can help achieve patient-therapist centered goals. With open-source applications readily available online, enormous quantities of information can be shared and utilized with the needs of various rehab professionals. Bermudez i Badia et al. are developing the Open Rehab Initiative that gives rehabilitation professionals a quick and easy way to access virtual programs for rehabilitation. It is an independent online portal that aims to help clinicians, scientists, engineers, game developers and end-users to interact with and share virtual rehabilitation tools. Along with the open-access of newly developed software, newer and more cost-effective VR headsets are being released. Integration of mobile devices along with some headsets have allowed for a cheaper alternative to the VR experience.

Some VR applications are being used to assist with assessment and treatment as a tool in rehabilitation [3] [4] [6]. To date, most VR systems primarily deliver visual and auditory feedback. Applications that are available allow individuals to experience a multitude of virtual environments and access to virtual avatars. These environments may simulate life situations that may include a virtual kitchen, a shopping isle, street crossing, tightrope walking, and virtual theme parks. Newer programs are being created that allow a person to manipulate virtual objects within a virtual world. Clinical psychologists and rehabilitation specialists use VR to provide new human-computer interactions [4]. This allows participants to engage in active participation within a computer-generated three-dimensional world rather than passive observers. Early research agrees that VR can assist with the facilitation of therapeutic change [3] [4]. This case report will follow closely with the integration of VR along with rehabilitation therapy in an outpatient vestibular physical therapy setting.

Those suffering from vestibular deficits commonly have complaints in the form of "dizziness". Some forms of dizziness include light-headedness or faintness, nausea, unsteadiness of one's feet, or vertigo. These symptoms can be caused by lesions anywhere in the vestibular pathway. Most cases are caused by peripheral disorders involving the inner ear, with central disorders of the brainstem or cerebellum being less common [7]. Those receiving treatment for 
these symptoms perform vestibular rehabilitation. Vestibular rehabilitation is an exercise-based treatment program designed to promote vestibular adaptation and substitution. Key exercises for vestibular rehabilitation are head-eye movements, maintaining balance with various orientations of head and trunk, repeating movements that provoke vertigo-like symptoms, and gradually exposing patients to various sensory and motor environments. The main goals for vestibular rehabilitation are to enhance gaze stability, enhance postural stability, improve vertigo, and to improve activities of daily living [8].

Increasing availability of VR technology and the creation of more interactive VR applications have helped integrate VR into today's society. VR technology provides a level of customization, control, and adaptability to virtual environments. VR offers the ability to create systematic human testing, training, and treatment environments. The variability of VR allows for sophisticated interactions, behavioral tracking, and performance recording. Similarly to vestibular rehabilitation, VR applications have the potential mimic movements and positions seen within the key exercises of vestibular rehabilitation.

A study by Kim et al. explores the link between smooth eye pursuits without head movement and its disruption to static balance [9]. Results of the study show a positive correlation with eye movements and disturbance of static equilibrium. This study implies the importance of gaze stability and oculomotor control in those suffering from balance deficits. In physical therapy, patients with central nervous system injuries may present with impairments with eye movements. Virtual environments can be used as a tool to assist in oculomotor exercises [9]. To objectively measure the amount of adaptation and habituation, the Dizziness Handicap Inventory (DHI), with minimal detectable change (MCD) of 18 , was used to quantify the patient's self-perception [10]. DHI has is valid and has high test-retest reliability for total score $(r=0.97, d f=12, p<0.0001)$, for sub-scale scores $(r=0.92-0.97, p<0.001)[11]$.

A study by McConville and Milosevic, suggests that active video games could be used to improve postural balance by challenging the vestibular system with various head movements [12] [13]. Some applications available include active video games that place a person into head positions that are seen in a vestibular rehabilitation program. Another study by Meldrum et al. tests the effectiveness of VR-based vestibular rehab compared to conventional rehab [14]. The results show that VR is a portable tool patients can take home and that therapists can use to record adherence and compliance to the program. To measure objective changes in balance, the Sensory Organization Test (SOT) was used. This outcome measure has an MDC of 8 points [15]. The SOT has adequate composite score reliability $($ ICC $=0.67)$ and has moderately high sensitivity $(85 \%)$ and specificity (77\%) in identifying vestibulopathies [15].

A randomized controlled trial by Kalron et al., examined the efficacy of a 6week VR balance training program for those with multiple sclerosis using the computer assisted rehabilitation environment (CAREN) system [16]. Results imply that the balance training based on the CAREN device is an effective me- 
thod of balance training for those with imbalance. The CAREN system shows that VR is capable of improving a patient's balance [16]. To measure improvements within functional balance, the Functional Gait Assessment (FGA) was performed. This measure has a MDC of 8 points [17]. The FGA has excellent test-retest reliability when administered by a student $(\mathrm{ICC}=0.80 ; 95 \% \mathrm{CI}=0.58$ - 0.91), excellent interrater reliability $(\mathrm{ICC}=0.84)$, and excellent internal consistency $(\alpha=0.79)[18]$.

A study by Maron et al. explores the physiological and behavioral indicators of anxiety during exposure in a VR environment [19]. In the study, participants who had acrophobia were immersed into a virtual cityscape at varying heights. Baseline measurements were recorded without VR, in VR at ground level, and in VR at height. The results showed that patients demonstrated the physiological and behavioral indicators when immersed in a virtual environment. A case study by Whitney et al. explores VR exposure therapy together with physical therapy to assist in the improvement of Acrophobia and pathological height vertigo [20]. These studies show a positive correlation between VR exposure therapy and decreased anxiety levels due to acrophobia. The virtual environment is so complex that it can trick the brain into perceiving a virtual object as real, triggering a physiological response. To get an objective measurement of this, the Activities-Specific Balance and Confidence Scale (ABCs) was used. This measure has a MDC of 12 [21]. The ABC has excellent test-retest reliability $(r=9.2, p<0.001)$, excellent internal consistency ( $\alpha=0.96$ ) [22] [23].

As current technologies continue to advance as well as continued research on VR, we will be one step closer to fully integrating virtual worlds into our everyday experiences. Current research has shown promise in the use of virtual environments and their effectiveness in the health care industry. As more low-cost hardware becomes readily available, it will be easier for VR to assimilate with society [3] [4]. Physical therapists can look forward to seeing VR as an assistive tool that will help provide a more efficient way to provide our patients the best treatment. The purpose of this case is to view the effectiveness of VR combined with traditional vestibular rehabilitation with a patient who poor postural control and a diagnosis of vestibular hypofunction.

\section{Case Description}

$\mathrm{AR}$ is a 50 -year-old male who presents to the clinic with a referral from his physician stating imbalance and dizziness following a motor vehicle accident (MVA) 6 weeks ago. The patient complained of blurry vision, ringing in the ears, and difficulty remembering things. He reports that symptoms increase with quick head movements and eases with rest. The patient has a history of asthma that is controlled. The patient has an endomorph body type and has not been very active outside of work. The patient works for a construction company which requires him to travel on-site and perform duties in high, elevated areas. He has a supportive family and likes to go out and spend time with his significant other and his daughter. The patient completed and signed an informed consent and 
information release form to be used as a subject in a case study.

An examination was performed that includes the patient history, a systems review, and test and measures to gather patient data as described in The Guide to Physical Therapy Practice [24]. The patient completed objective measures the Dizziness Handicap Scale (DHI) Activities-Specific Balance Confidence Scale (ABCs), Functional Gait Assessment (FGA), Sensory Organization Test (SOT), and Motor Control Test (MCT). The DHI and ABCs are surveys that were designed to objectively measure the patient's subjective history of their ongoing impairments. The patient scored a 74 on the DHI which places him in the category of severe vestibular dysfunction [25]. With the ABCs, he scored a $75 \%$ which places him within normative values at his age range [22]. Upon gathering history, the patient described his dizziness as a swaying sensation as if he was "riding a boat." He stated a feeling of disequilibrium and dizziness lasting between 10 seconds to 30 seconds. Ease of symptoms occurred with rest and with his eyes closed. The patient reported not taking any medications. During the physical examination, the patient presented with a presence of saccadic eye movements during smooth-purist movements and complained of an increase in dizziness symptoms. The patient demonstrated a loss of vergence of the left (L) eye at $15 \mathrm{~cm}$. A Head Impulse Test (HIT) was performed and the patient demonstrated a positive HIT on his L side. The patient performed full cervical range of motion (ROM) without pain. His lower extremity strength was within normal limits. Functional tests, FGA, SOT, and MCT, were used to give the physical therapist a better understanding how the patient moves within certain conditions. The patient scored a 21/30 with the FGA which places him bellow the normative values at his age [26]. A NeuroCom SMART Balance Master ${ }^{\oplus}$ device was available to assist in performing the SOT and MCT. SOT revealed a vestibular dysfunction and gave a composite score of 67 which placed him below the normative values at his age [27]. For MCT, the patient received a composite score of 142 which places him within normal values at his age [27]. Reflexes and sensation were normal and intact. No radiographic images were provided.

\section{Patient Evaluation}

The patient presents with a composite impairment of decreased balance. Indirect impairments include increased dizziness, blurry vision, and tinnitus. Secondary impairments include disequilibrium and gait disturbances. With the given information, it is suspected that the patient has a diagnosis of unilateral L sided vestibular hypofunction. Since the patient explained an increase of symptoms with quick head movements, a differential diagnosis of benign paroxysmal positional vertigo (BPPV) was explored. The patient demonstrated a pain-free cervical range of motion (ROM). The patient's description of symptoms is not consistent with symptom presentation of BPPV and did not present with nystagmus during the Dix Hallpike. A positive HIT suggests the presence of a unilateral peripheral vestibular weakness on the L side [28]. The DHI also supports this hypothesis and places the patient in the category of severe vestibular dysfunction 
[25]. Scores from SOT further support of a vestibular deficit. The ABCs and MCT reveal that the patient is a decreased risk of sustaining a fall. Values of FGA, broken up in Table 1, suggest that the patient with a mild balance impairment. The patient demonstrated noted difficulties with gait with horizontal head turns, gait with a narrow base of support, gait with eyes closed and backward ambulation.

According to the International Classification of Functioning, Disability, and Health (ICF) [29], the patient activity limitations include ambulation and reading for longer than 30 minutes. Participation limitations include the inability to perform job requirements, driving long distances, enjoying theme parks with his family. Based on these limitations, the patient received a physical therapy diagnosis of R26.81 Unsteadiness on feet and R42 Dizziness and Giddiness.

\section{Plan of Care}

Following the physical therapy examination and evaluation, a plan of care was discussed with the patient and short- and long-term goals were established. The patient agreed to being seen two to three times a week for eight weeks with 40-minute sessions at an outpatient vestibular setting [30] [31]. Short-term goals were to be accomplished in the four weeks which included: decreasing DHI score to 50 on DHI and increasing ABCs score to $82 \%$. The primary focus within the first four weeks was to improve his acute unilateral vestibular hypofunction and to prepare him for more complex challenges with VR [31]. Clinical practice guidelines recommend that patients with acute unilateral vestibular hypofunction should have supervised therapy for two to three weeks [31]. Research has shown that patients suffering from vestibular deficits may require up to 8 weeks of vestibular therapy before seeing significant improvement [30]. Long-term goals expected to be accomplished by discharge, included: an increase in ABCs scores to $>92 \%$, improvement of FGA score $>26$, improved SOT composite score $>75$, and a negative HIT.

Table 1. FGA scores.

\begin{tabular}{ccc}
\hline & T1 & T3 \\
\hline Gait on Level Surface & 2 & 3 \\
Change in Gait Speed & 2 & 3 \\
Gait with Horizontal Head Turns & 2 & 3 \\
Gait with Vertical Head Turns & 2 & 3 \\
Gait and Pivot Turn & 3 & 3 \\
Step over Obstacle & 3 & 3 \\
Gait with Narrow Base of Support & 2 & 3 \\
Gait with Eyes Closed & 1 & 3 \\
Ambulating Backwards & 1 & 2 \\
Steps & 3 & 3 \\
Total Score & 21 & 29 \\
\hline
\end{tabular}

T1-Initial evaluation, T3-Discharge. 
The patient's prognosis was good to achieve both short and long-term goals as he demonstrated high levels of motivation, a supportive family, and a minimally active lifestyle. Early treatment strategies included vestibular exercises, balance training, and gait training [9] [31] [32]. At follow-up, the patient was introduced to a VR environment. VR has shown promise with the psychological as well as physical aspects of rehabilitation [4] [14] [19]. The purpose for VR was to challenge his balance systems further and incorporate new strategies to enable him return to full-time work status.

\section{Implementation of Interventions/Re-Assessment}

Interventions utilized during the patient's therapy focused on improving vestibular function to improve overall balance [9] [32]. The patient was cleared of any cervical restrictions that may impede vestibular exercises. The patient had no complaints of neck pain, focal neck tenderness or spasms, or any neurological deficits. The patient was educated on the importance of maintaining a consistent exercises program and that he would see significant changes in his overall balance for approximately 8 weeks [30]. With exercises and activities, the patient was placed into a harness which was attached to a ceiling track to prevent a fall. A supervised student physical therapist was present to assist the patient if he were to experience a loss of balance.

During the first four weeks, the patient engaged in vestibular rehabilitation therapy [8] [31]. Exercises performed were to improve gaze stability, enhance eye movements, increase postural stability, decrease vertigo, and improve activities of well-being [8]. To enhance gaze stability and to improve vestibule-ocular reflex, the patient performed $\mathrm{x} 1$ viewing [8]. Progressions to each exercise are described in Table 2 and were progressed as needed. These progressions involved changing the eyes, head movement, speed, movement amplitude, target location, target distance, foot position, base hardness, base width, and gait [8]. Other adaptation exercises included quick movements between two targets and smooth pursuits. Progressions were done in a similar fashion with the other exercises mentioned above. The patient was educated to delete on the principles of vestibular adaptation and habituation. The patient was instructed to perform gaze stability exercises four to five times daily for a total of 20 - 40 minutes a day and at least 20 minutes of posture and gait stability exercises [8]. The goals of postural stability exercises were to help the patient learn to use stable visual references and surface somatosensory information from their primary postural sensory system, use the remaining vestibular function, identify efficient and effective alternative postural movement strategies, and recover normal postural strategies [8]. The patient also received a home exercise program (HEP) to supplement the exercises performed within the clinic. He was instructed to perform his adaptation exercises two to three times a day for a total of 20 minutes a day and to perform at least 20 minutes of posture stability HEP exercises [8] [31]. At the beginning of each consequent therapy session, the patient was asked if he performed his exercises and was asked to demonstrate the activities to ensure compliance with the program. 
Table 2. Exercises [8] [30].

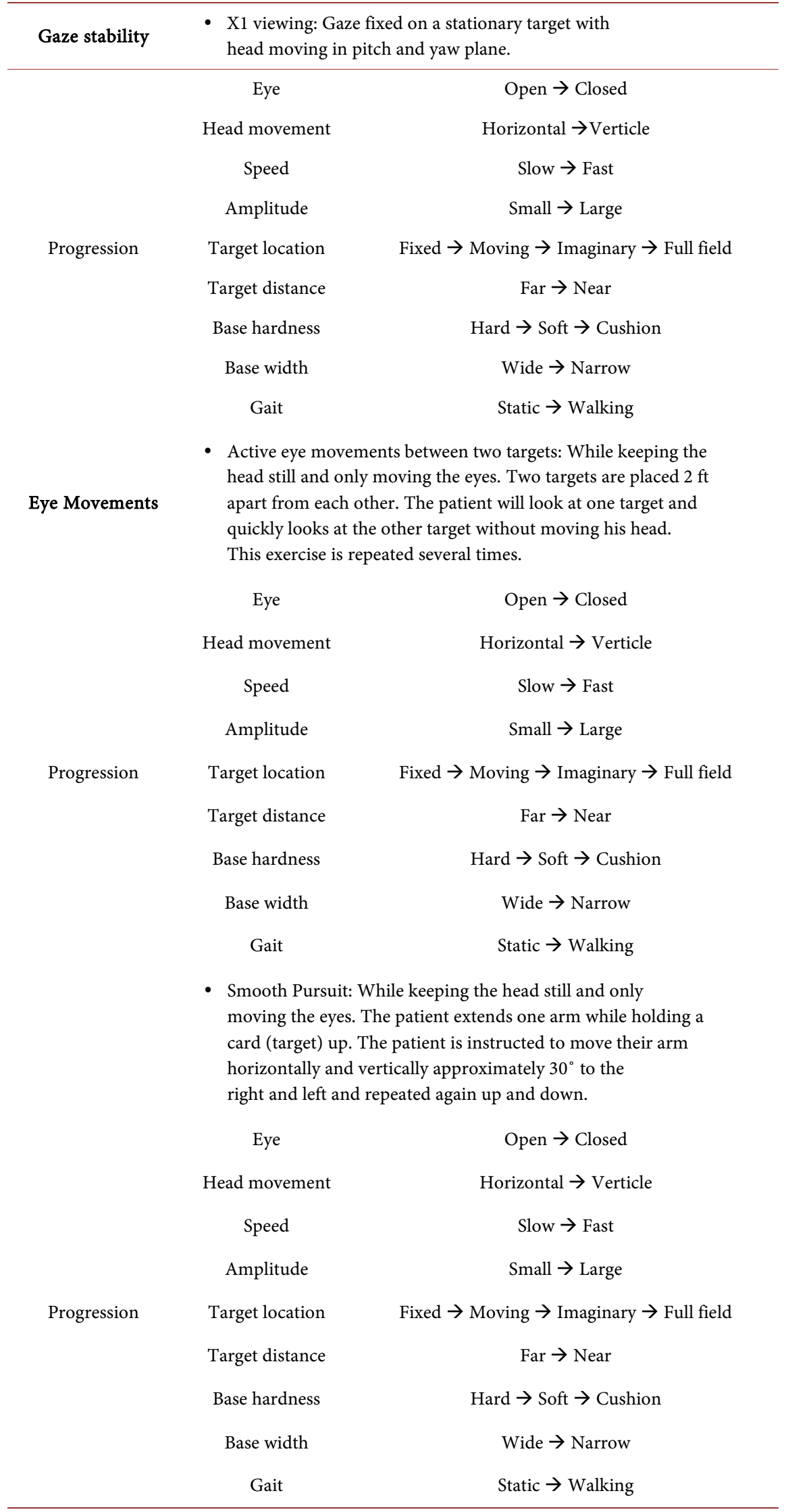




\section{Continued}

\begin{tabular}{ccc}
\hline Postural Stability & Balance: Held for 30 second intervals. \\
Eye & Open $\rightarrow$ Closed \\
Speed & Slow $\rightarrow$ Fast \\
Amplitude & Small $\rightarrow$ Large \\
Progression & Foot position & Shoulder width $\rightarrow$ Together $\rightarrow$ Tandum $\rightarrow$ Single leg \\
& Arm position & Outstretched $\rightarrow$ Close to body $\rightarrow$ Across chest \\
& Base hardness & Hard $\rightarrow$ Soft $\rightarrow$ Cushion \\
Base width & Wide $\rightarrow$ Narrow \\
Gait & Static $\rightarrow$ Walking \\
\hline
\end{tabular}

At the 10th visit, the patient was reassessed. There were mild improvements in his FGA $(F G A=23)$ and $A B C s(A B C=80)$, although this score was not significant enough to claim a minimal detectable change. This further supports the article by Lee HJ [30], indicating that objective vestibular function and vestibular compensation will improve with eight weeks of continuous vestibular rehabilitation. There was a significant change in the patient's DHI $(\mathrm{DHI}=50)$ score at the 10th visit. The patient reported of having decreased dizziness intensity, frequen$\mathrm{cy}$, and duration. At this point in time, the patient was participating in part-time stats at work. He also felt comfortable reading for 30 minutes and was excited to continue his improvement.

Following his follow-up, the patient was introduced to a virtual reality (VR) environment [3] [4] [14]. The goal for VR was continued challenges to his oculomotor and vestibular system with the intent to return to full-time work. The patient performed VR activities for 20 - 30 minutes and was instructed to inform the SPT if there were any changes in symptoms [14] [31]. The VR applications that were used included: Vanguard V, InMind VR, InCell VR, VR TunnelRace, Roller Coaster, and The Walk VR. Some of these applications were used with the idea that they were able to mimic movements and activities used in a VRT [12]. Vanguard V, InMind VR, InCell VR, and VR Tunnel Race were games that had the patient maintain static balance while dynamically moving his head into various head positions (pitch, yaw, and roll). These games mimic many of the movements that the patient performs during therapy. Each game has a mixture of optokinetic exercises with vestibular exercises because the game encourages head movement with a busy field of view [4]. VR also gave the SPT an opportunity to provide variety to the patient's program. Another objective of the VR environment was decreasing the patient's anxiety with heights that may simulate experiences in his line of work [13]. The Roller Coaster and The Walk VR apps were utilized to decrease the patient's anxiety with heights following similarities of exposure therapy [19]. The Roller Coaster app is an application where a person is placed in a first-person view of a roller coaster ride. The quick movements and changing directions of simulator were used to help improve the patient's visual systems. The Walk VR is a simulation of a person walking a tightrope be- 
tween the Twin Towers in New York. The patient began these activities on a firm surface and was progressed to varying surfaces and different stance positions. Due to the restrictions of the applications and concerns of safety, the patient remained static throughout all VR simulations.

\section{Outcomes}

At discharge, the patient reported feeling overall improvement since his initial evaluation. Improvements were seen in DHI, ABC, FGA, and SOT scores when compared to his initial evaluation. See Table 1 and Table 3 for more details. There were noticeable improvements during the performance of the FGA. The patient no longer demonstrated difficulties with walking with head movements, walking with a narrow base of support, and walking with eyes closed. Although there were improvements in backward ambulation, the patient revealed some difficulty. He was able to achieve all short and long term goals estimated during the plan of care. The patient expressed enthusiasm as to the improvement he achieved through his time in therapy. He described a significant decrease in the intensity, duration, and frequency of his dizziness symptoms since his evaluation.

The patient also had improvements in ICF model of activity and participation levels. For activity, the patient was able to read a book and his phone over an hour without reproduction of symptoms. He was able to demonstrate a normal gait pattern without a loss of balance or any deviations. His participation improved as he was able to return to work with full confidence. The patient also explained that he was able to drive for over 2 hours without any reproduction of symptoms. He described that he was able to participate in more family functions without any worries of losing his balance or an increase in dizziness episodes. $\mathrm{He}$ mentioned that the last time he had an episode of dizziness was two weeks ago when he was driving through a mountain pass. He explained that he was able to implement the activities he learned from therapy to quickly resolve any episodes of dizziness. It was recommended that the patient continue performing his vestibular exercises of $\times 1$ viewing and smooth pursuits 3 times a day for a total of 20 - 40 minutes to help maintain optimal function [31].

\section{Discussion}

The purpose of this paper is to explore the use of VR as a viable tool for a balance program for a patient who is experiencing visual and vestibular impairments. The patient had improved ABCs scores from $75 \%$ to $95 \%$ at the time of

Table 3. DHI, ABC, SOT scores.

\begin{tabular}{cccc}
\hline & T1 & T2 & T3 \\
\hline DHI & 74 & 50 & 4 \\
ABC & $75 \%$ & $80 \%$ & $95 \%$ \\
SOT & 67 & - & 80 \\
\hline
\end{tabular}

$\mathrm{T} 1$ - Initial evaluation, $\mathrm{T} 2-10^{\text {th }}$ visit follow up, T3-Discharge. 
discharge. The ABCs is an outcome measure where the patient rates their balance confidence in performing activities. The patient was able to change with an increase of 20 points to his initial evaluation. This demonstrates that the patient has more confidence in his balance with the help of VR and VRT. Research has shown that psychological factors such as anxiety from fear of falling are linked with decreased confidence with balance [19] [20] [33]. It can be inferred that the patient's confidence in performing activities increased as the patient's anxiety decreased while performing within VR.

Other studies have shown the benefits of a vestibular rehabilitation program in patients with balance deficits due to visual, somatosensory, and vestibular deficits [8] [30]. We have explored research regarding the use of VR technology to compliment exercises and activities within a vestibular rehabilitation program. These include gaze stability and smooth-pursuit eye movements as well as head positions achieved through various applications that are available [9] [12] [13]. The patient showed steady improvement when measured from baseline to his follow-up visit with DHI scores improving from 74 to 50 . Following the application of VR into his rehabilitation program, his DHI scores continued to improve from 50 to 4 at discharge. DHI scores placed the patient from a severe vestibular dysfunction to a mild vestibular dysfunction. This supports research of active head movements within vestibular rehabilitation as well as within the VR environment [12] [13] [14]. Activity improvements were seen through his ability to drive longer than two hours without increased dizziness symptoms, being able to read for over one hour without a subjective increase in symptoms, and demonstrated the ability to walk down a hallway without walking into an object. The $\mathrm{ABCs}$ and $\mathrm{DHI}$ are a subjective assessment done by the patient.

Improvements in these psychological areas are transferred functionally and were assessed using the SOT and FGA. Initially, the patient received a composite score of 67 with deficits seen within the visual and vestibular systems. At discharge, the patient received a composite score of 80 with all of his balance systems falling within the normative values of those at his age. With FGA testing, the patient initially received a score of 21 which placed the patient below normative values indicating that he was a falls risk. At time of discharge, the patient scored a 29/30. This shows that the patient had a significant change in scores from initial evaluation to discharge. These increases in scores support research regarding the use of VR as a supportive tool for vestibular rehabilitation [14]. With these improvements, the patient was able to return to full-time status with his work and was able to participate in more family activities.

Clinical findings of this case support the use of VR as a potential tool in the treatment of patients suffering from balance deficits due to visual and vestibular deficits. This case has shown the effectiveness of VR in improving a patient's confidence, vestibular function, and balance systems. These findings are significant, as there is limited research that is specific to vestibular rehabilitation and the use of virtual environments. As more VR technologies are being developed and become low-cost, it will be available for anyone to use [3] [4]. More research 
will be needed to see the effectiveness of VR in the assistance in evaluating and treating patients.

Some limitations of this study include the technology available for this case. The accessories that were used in the case were a Vigica VR headset and an iPhone S5. Many of the applications used throughout each therapy session were free apps that were available on the iTunes App Store. There were limitations of control the therapist had as the patient performed his exercises in the virtual environment. Since many applications required a fee to gain access, there were difficulties in gearing task-specific activities to help the patient achieve their long-term goals. Another limitation with this particular set-up is the patient's ability to interact with his virtual environment. With the current technology being used, the patient mostly played a passive role within VR. Some of the activities the patient engaged in placed the patient in a position where the patient can only look around as a video played. In more interactive video games, the patient can move their head and stare at an object for a certain time to perform an action or move their head to move the virtual avatar within the virtual world. The headset itself can be considered another limitation. Due to the size and added weight of the headset, there may be minor imbalances created as the patient was wearing it. With further development of VR technologies, we will gain access to more task-specific activities, improved control throughout various virtual environments, more cost-effective equipment, and more efficient ways to provide essential feedback.

An alternative explanation to the patient's improvements may be due to his maintained compliance to his vestibular rehabilitation program. A study by Lee explains how vestibular rehabilitation may take up to 8 weeks before significant improvements show [30]. Following the 4th week of the patient's therapy, the patient was introduced to VR activities on top of performing his usual vestibular exercises.

Areas for improvement include the patient performing VR activities alone to see the effectiveness of VR. It would have also been helpful if the patient was able to buy a VR headset so that he could perform VR exercises at home. If the clinic had better access to an improved VR experience, it would allow for greater control of activities the patient has engaged it. It will also enable the therapist to see what the patient sees which allows the therapist to provide various types of feedback to improve motor learning [34]. Another weakness was that this case did not fully follow the clinical practice guidelines. Clinical practice guidelines explain that patients suffering from an acute unilateral vestibular hypofunction will see improvements in vestibular function when receiving supervised vestibular rehabilitation therapy once a week for about 3 weeks along with a HEP [31]. More time was required for treatment since the patient did not feel he has reached his goals of therapy due to continued imbalance and gait deviations. A virtual environment was used to continue challenging his vestibular and visual systems so the patient would be able to return to full-time work status. The virtual world gave the therapist the ability to virtually simulate the patient's work 
environment and to build the patient's confidence [19]. Improved VR technology would give the therapist the ability to check for adherence of the patient's HEP and allows the therapist to give more constructive feedback to their patient to improve scores [4]. As technology continues to advance, there will be improved control of variables within VR which can create a better immersive experience for all who participate [4]. Future research will be needed to see how VR stands to vestibular rehabilitation alone or both combined. A study like this will allow us to see if VR would work with vestibular rehabilitation as an adjunct activity.

In summary, the patient demonstrated significant improvements with vestibular rehabilitation and VR activities as evidenced by improved scores in ABCs, DHI, SOT, and FGA. Some of these applications allow for task-specific activities to allow the patient to engage in more meaningful interactions. The patient also expressed increased confidence in performing gait activities, returning to work, and engaging in more family activities at home. These improvements can be attributed to the combination of vestibular rehabilitation and VR exercises. This case report provides valuable information regarding the use of VR as an intervention technique to improve postural balance in individuals suffering from visual deficits and unilateral vestibular hypofunction impairments.

\section{References}

[1] Chaudhry, B., Wang, J., Wu, S., Maglione, M., Mojica, W., Roth, E., Morton, S.C. and Shekelle, P.G. (2006) Systemic Review: Impact of Health Information Technology on Quality, Efficiency, and Costs of Medical Care. Annals of Internal Medicine, 114, 742-752. https://doi.org/10.7326/0003-4819-144-10-200605160-00125

[2] Definition of Virtual Reality. Merriam-Webster. https://www.merriam-webster.com/dictionary/virtual\%20reality

[3] Weiss, P.L. and Katz, N. (2004) The Potential of Virtual Reality for Rehabilitation. Journal of Rehabilitation Research \& Development, 41, 7-10.

[4] Rizzo, A. and Kim, G.J. (2005) A SWOT Analysis of the Field of VR Rehabilitation and Therapy. Presence, 14, 119-146. https://doi.org/10.1162/1054746053967094

[5] Riva, G. and Wiederhold, B.K. (2015) The New Dawn of Virtual Reality in Health Care: Medical Simulation and Experimental Interface. Annual Review of CyberTherapy and Telemedicine, 13, 3-6.

[6] Deustch, J.E. (2016) Open Rehabilitation Initiative: Design and Formative Evaluation.

[7] Blumenfeld, H. (2010) Neuroanatomy through Clinical Cases. 2nd Edition, Sinauer Associates, Inc.

[8] Han, B.I., Song, H.S. and Kim, J.S. (2011) Vestibular Rehabilitation Therapy: Review of Indications. Journal of Clinical Neurology, 7, 184-196. https://doi.org/10.3988/jen.2011.7.4.184

[9] Kim, S.-Y., Moon, B.-Y. and Cho, H.G. (2016) Smooth-Pursuit Eye Movements without Head Movement Disrupt the Static Body Balance. Journal of Physical Therapy Science, 28, 1335-1338. https://doi.org/10.1589/jpts.28.1335

[10] Cohen, H.S. and Kimball, K.T. (2003) Increased Independence and Decreased Vertigo after Vestibular Rehabilitation. Otolaryngology_Head and Neck Surgery, 128, 60-70. 
[11] Tamber, A.-L., Wilhelmsen, K.T. and Strand, L.I. (2009) Measurement Properties of the Dizziness Handicap Inventory by Cross-Sectional and Logitudinal Designs. Health and Quality of Life Outcomes, 7, 1-16. https://doi.org/10.1186/1477-7525-7-101

[12] McConville, K.M.V. and Milosevic, M. (2014) Active Video Game Head Movement Inputs. Personal and Ubiquitous Computing, 18, 253-257. https://doi.org/10.1007/s00779-013-0662-2

[13] McConville, K.M.V., Virk, S. and Milosevic, M. (2007) Head Movement Effects in a Cost-Effective Virtual Reality Training Environment for Balance Rehabilitation. Virtual Rehabilitation, Venice, 27-29 Sept. 2007, 87. https://doi.org/10.1109/ICVR.2007.4362143

[14] Meldrum, D., Herdman, S., Moloney, R., Murray, D., Duffy, D., Malone, K., French, H., Hone, S., Conroy, R. and McConn-Walsh, R. (2012) Effectiveness of Conventional versus Virtual Reality Based Vestibular Rehabilitation in the Treatment of Dizziness, Gait and Balance Impairment in Adults with Unilateral Peripheral Vestibular Loss: A Randomised Controlled Trial. BMC Ear, Nose and Throat Disorders, 12, 1-8. https://doi.org/10.1186/1472-6815-12-3

[15] Wrisley, D.M., Stephens, M.J., Mosley, S., Wojnowski, A., Duffy, J. and Bukard, R. (2007) Learning Effects of Repetitive Administrations of the Sensory Organization Test in Healthy Young Adults. Archives of Physical Medicine and Rehabilitation, 88, 1049-1054.

[16] Kalron, A., Fonkatz, I., Frid, L., Baransi, H. and Achiron, A. (2016) The Effect of Balance Training on Postural Control in People with Multiple Sclerosis Using the CAREN Virtual Reality System: A Pilot Randomized Controlled Trial. Journal of NeuroEngineering and Rehabilitation, 13, 1-10. https://doi.org/10.1186/s12984-016-0124-y

[17] Marchetti, G.F., Lin, C.-C., Alghadir, A. and Whitney, S.L. (2014) Responsiveness and Minimal Detectable Change in Dynamic Gait Index and Functional Gait Index in Persons with Balance and Vestibular Disorders. Journal of Neurologic Physical Therapy, 38, 119-124. https://doi.org/10.1097/NPT.0000000000000015

[18] Leddy, A.L., Crowner, B.E. and Earhart, G.M. (2011) Functional Gait Assessment and Balance Evaluation System Test: Reliability, Validity, Sensitivity, and Specificity for Identifying Individuals with Parkinson Disease Who Fall. Physical Therapy, 91, 102-113. https://doi.org/10.2522/ptj.20100113

[19] Powell, V. (2016) Differential Effect of Neutral and Fear-Stimulus Virtual Reality Exposure on Physiological Indicators of Anxiety and Acrophobia.

[20] Whitney, S.L., Jacob, R.G., Sparto, P.J., Olshansky, E.F., Detweiler-Shostak, G., Brown, E.L. and Furman, J.M. (2005) Acrophobia and Pathological Height Vertigo: Indications for Vestibular Physical Therapy? Physical Therapy, 85, 443-458.

[21] Steffen, T. and Seney, M. (2008) Test-Retest Reliability and Minimal Detectable Change on Balance and Ambulation Tests, the 36-Item Short-Form Health Survey, and the Unified Parkinson Disease Rating Scale in People with Parkinsonism. Physical Therapy, 88, 733-746.

[22] Huang, T.-T. and Wang, W.-S. (2009) Comparison of Three Established Measures of Fear of Falling in Community-Dwelling Older Adults: Psychometric Testing. International Journal of Nursing Studies, 46, 1313-1319.

[23] Powell, L.E. and Myers, A.M. (1995) The Activities-Specific Balance Confidence (ABC) Scale. The Journals of Gerontology. Series A, Biological Sciences and Medical Sciences, 50A, M28-M34. https://doi.org/10.1093/gerona/50A.1.M28

[24] American Physical Therapy Association (2016) Guide to Physical Therapy Practice 
3.0. APTA. http://guidetoptpractice.apta.org/content/current

[25] Whitney, S.L., Wrisley, D.M., Brown, K.E. and Furman, J.M. (2004) Is Perception of Handicap Related to Functional Performance in Persons with Vestibular Dysfunction. Otology \& Neurotology, 25, 139-143. https://doi.org/10.1097/00129492-200403000-00010

[26] Walker, M.L., Austin, A.G., Banke, G.M., Foxx, S.R., Gaetano, L., Gardner, L.A., McElhiney, J., Morris, K. and Penn, L. (2007) Reference Group Data for the Functional Gait Assessment. Physical Therapy, 87, 1468-1477.

[27] Whitney, S.L., Marchetti, G.F. and Schade, A.I. (2006) The Relationship between Falls History and Computerized Dynamic Posturography in Persons with Balance and Vestibular Disorders. Archives of Physical Medicine and Rehabilitation, 87, 402-407.

[28] Rehabilitation Institute of Chicago (2017) Head Impulse Test. http://www.rehabmeasures.org/Lists/RehabMeasures/DispForm.aspx?ID=1196

[29] Stucki, G. (2005) International Classification of Functioning, Disability, and Health (ICF): A Promising Framework and Classification for Rehabilitation Medicine. American Journal of Physical Medicine \& Rehabilitation, 84, 733-740. https://doi.org/10.1097/01.phm.0000179521.70639.83

[30] Lee, H.J. and Choi-Kwon, S. (2016) Effectiveness of Self-Efficacy Promoting Vestibular Rehabilitation Program for Patients with Vestibular Hypofunction. Journal of Korean Academy of Nursing, 46, 710-719. https://doi.org/10.4040/jkan.2016.46.5.710

[31] Hall, C.D., Herdman, S., Whitney, S.L., Cass, S.P., Clendaniel, R.A., Fife, T.D., Furman, J.M., Getchius, T.S.D., Goebel, J.A., Shepard, N.T. and Woodhouse, S.N. (2016) Vestibular Rehabilitation for Peripheral Vestibular Hypofunction: An Evidence-Based Clinical Practice Guideline. American Physical Therapy Association Neurology Section, 40, 123-155. https://doi.org/10.1097/NPT.0000000000000120

[32] Scheltinga, A., Honegger, F., Timmermans, D.P.H. and Allum, J.H.J. (2016) The Effect of Age on Improvements in Vestibulo-Ocular Reflexes and Balance Control after Acute Unilateral Peripheral Vestibular Loss. Frontiers in Neurology, 7, 18. https://doi.org/10.3389/fneur.2016.00018

[33] Young, W.R. and Williams, A.M. (2015) How Fear of Falling Can Increase Fall-Risk in Older Adults: Applying Physiological Therapy to Practice Observations. Gait \& Posture, 41, 7-12.

[34] Sattelmayer, M., Elsig, S., Hilfiker, R. and Baer, G. (2016) A Systematic Review and Meta-Analysis of Selected Motor Learning Principles in Physiotherapy and Medical Education. BMC Medical Education, 16, 1-22.

https://doi.org/10.1186/s12909-016-0538-z 
Submit or recommend next manuscript to SCIRP and we will provide best service for you:

Accepting pre-submission inquiries through Email, Facebook, LinkedIn, Twitter, etc. A wide selection of journals (inclusive of 9 subjects, more than 200 journals)

Providing 24-hour high-quality service

User-friendly online submission system

Fair and swift peer-review system

Efficient typesetting and proofreading procedure

Display of the result of downloads and visits, as well as the number of cited articles Maximum dissemination of your research work

Submit your manuscript at: http://papersubmission.scirp.org/

Or contact ijcm@scirp.org 